\title{
Vesicular ATP-binding cassette transporters in human disease: relevant aspects of their organization for future drug development
}

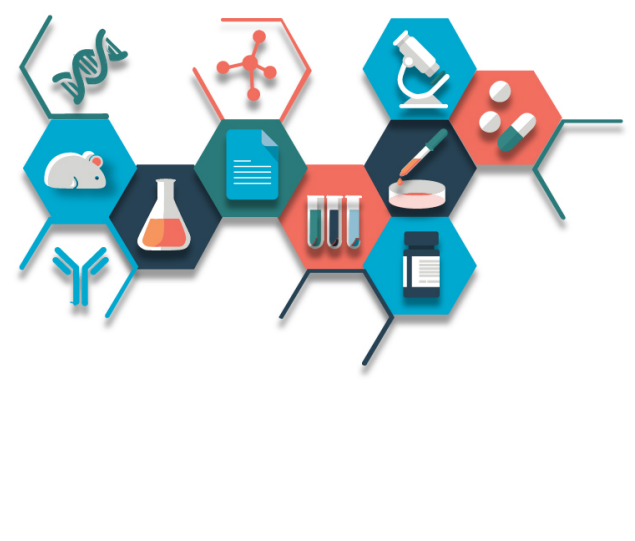

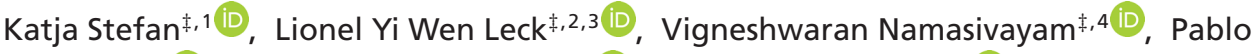 \\ Bascuñana1 (iD), Michael Li-Hsuan Huang ${ }^{5}$ (D) Patrick Johannes Riss ${ }^{6}$ (D) Jens Pahnke ${ }^{1,7,8,9}$ (iD), \\ Patric Jan Jansson*,2,3 (iD) \& Sven Marcel Stefan**,1,2,4,6 (iD) \\ ${ }^{1}$ Department of Neuro-/Pathology, University of Oslo \& Oslo University Hospital, Oslo, Norway \\ ${ }^{2}$ Cancer Drug Resistance \& Stem Cell Program, School of Medical Science, Faculty of Medicine \& Health, University of Sydney, \\ Sydney, New South Wales, Australia \\ ${ }^{3}$ Bill Walsh Translational Cancer Research Laboratory, Kolling Institute of Medical Research, Royal North Shore Hospital, Northern \\ Clinical School, Faculty of Medicine \& Health, University of Sydney, Sydney, New South Wales, Australia \\ ${ }^{4}$ Department of Pharmaceutical \& Cellbiological Chemistry, University of Bonn, Bonn, Germany \\ ${ }^{5}$ Discipline of Pathology, Faculty of Medicine \& Health, University of Sydney, Sydney, New South Wales, Australia \\ ${ }^{6}$ Department of Chemistry, University of Oslo, Oslo, Norway \\ ${ }^{7}$ LIED, University of Lübeck, Lübeck, Germany \\ ${ }^{8}$ Department of Pharmacology, Faculty of Medicine, University of Latvia, Riga, Latvia \\ ${ }^{9}$ Department of Bioorganic Chemistry, Leibniz-Institute of Plant Biochemistry, Halle, Germany \\ *Author for correspondence: patric.jansson@sydney.edu.au \\ **Author for correspondence: s.m.stefan@medisin.uio.no \\ $¥$ Authors contributed equally
}

"besides $A B C$ transporters themselves, the vesicles in which they reside, the lipid raft microdomains in which they are organized and oligomerized as well as the cytoskeleton are new potential drug targets."

First draft submitted: 24 September 2020; Accepted for publication: 29 September 2020; Published online: 26 October 2020

$\mathrm{ABC}$ transporters in disease: an introduction

ATP-binding cassette $(\mathrm{ABC})$ transporters are membrane-bound proteins that actively translocate endo- and xenobiotic solutes across cellular membranes at the expense of energy by ATP hydrolysis, often against a concentration gradient [1]. In disease, these transporters are often altered in expression and/or functional activity. The multidrug resistance (MDR) phenotype in cancer, for example, occurs due to the overexpression of these transport proteins. Here, $\mathrm{ABC}$ transporters confer cross-resistance to structurally and functionally diverse and chemically unrelated antineoplastic agents [1,2]. Virtually every single type of the $49 \mathrm{ABC}$ transporters confers resistance to antineoplastic agents, with many of them having a broad substrate range [2]. Strikingly, certain representatives (e.g., ABCB1, $\mathrm{ABCC} 1$ and $\mathrm{ABCG} 2$ ) have an overlapping substrate range [1,2], which enables these transporters to compensate for the functional loss of their siblings after selective inhibition and/or downregulation. On the other hand, some transporters have a differing substrate range, leading to a universal MDR phenotype, covering almost the whole range of first- and second-line anticancer agents when co-expressed. The overlapping substrate range and regulatory compensation of $\mathrm{ABC}$ transporters may be one major aspect why clinical studies with selective $\mathrm{ABC}$ transporter inhibitors have failed [1].

Another area where ABC transporters have been shown to play an important role is Alzheimer's disease (AD) [37]. $\mathrm{AD}$ is a neurodegenerative disorder which is characterized by the pathological intracerebral precipitation of extracellular $\beta$-amyloid (A $\beta$ ) protein plaques [3]. These neurotoxic $A \beta$ proteins contribute to a decline of synaptic communication, neuronal atrophy, cognitive impairments and death after long-term accumulation and exposure 
to neurons [3]. ABC transporters were discovered as key proteins in the process of production, degradation and clearance of $A \beta$ proteins [3-7]. Remarkably, the very same representatives that have a bad reputation in cancer-related MDR - ABCB1, ABCC1 and ABCG2 - are associated with (Direct) $A \beta$ protein export from the brain to the blood stream across the blood-brain barrier (BBB) [1]. However, many other ABC transporters have been linked to the pathogenesis of $\mathrm{AD}$, especially (phospho)lipid and cholesterol transporters (e.g., ABCA1-2, ABCA5, ABCA7, ABCG1-2 and ABCG4) [3]. Similar to cancer-related MDR, the above-mentioned interrelationship between $A B C$ transporter function and compensatory expression applies to $A D$ as well, which has been demonstrated for certain representatives (e.g., ABCB1 or ABCG2) [3]. However, the intricate relationship between the up- and downregulation of $\mathrm{ABC}$ transporters in both cancer-related MDR and $\mathrm{AD}$ is still poorly understood and potentially underrated.

Both examples, cancer-related MDR and $\mathrm{AD}$, represent the $\mathrm{ABC}$ transporter-related dogma of a 'static' frontier, where the blood-tumor barrier (BTB) as well as the BBB are commonly believed as their major 'venue' of action. However, several reports have already identified $\mathrm{ABC}$ transporters as participants in rare lysosomal storage disorders, such as Fabry disease (ABCB11 and ABCC5 [8]) and Nieman-Pick type C disease (ABCG5 and ABCG8 $[9,10]$ ) and a huge body of evidence underlines that subcellular(-originating) structures as carriers of $\mathrm{ABC}$ transporters may play an essential role in major $\mathrm{ABC}$ transporter-related diseases, such as cancer-related MDR or AD.

\section{Subcellular ABC transporters: function beyond logistics}

It is crucial to differentiate between subcellular logistics and subcellular function of $\mathrm{ABC}$ transporters. There is no doubt that all $49 \mathrm{ABC}$ transporters are native intracellular proteins that are produced, processed and distributed during their synthesis in the endoplasmic reticulum [11], post-translational modification in the Golgi apparatus [11], pooling and/or sorting in the post-/trans-Golgi network [12,13], and trafficking, sorting and/or recycling in vesicular bodies [11]. And yet, this is all of logistical nature and the fusion and fission of endosomal and endocytic vesicles with the plasma membrane as well as with one another as part of the above mentioned steps is accompanied by a rather temporary residency of the individual transporter within the respective step - implying no additional function except for its relocation.

Nevertheless, many ABC transporters have (sometimes in addition to their lamellar localization and function) an intracellular purpose and target ab initio (multi)vesicular bodies and organelles inside the cell as a real subcellular destination rather than an intermediate 'stopover'. These destinations include [early/late (acidic)] endo- or lysosomes (e.g., ABCA4 [14], ABCA5 [15], ABCB1 [11], ABCB6 [16], ABCB9 [17], ABCC4-5 [18,19], ABCD4 [20], ABCG1 [21,22] or ABCG4 [22]), proteasomes (e.g., ABCB9 [17]), mitochondria (e.g., ABCB7-8 [16], ABCB10 [16], ABCC1 [23] or ABCC6 [24]), peroxisomes (e.g., ABCD1-3 [20]) or phago(lyso)somes (ABCA4 [14] or ABCF1 [25]). Beyond their regular (sub)cellular function, it has also been established that (re)localization of $A B C$ transporters into intracellular vesicles may cause, or at least contribute to, human diseases. Regarding cancer-related MDR, several $\mathrm{ABC}$ transporters were proven to sequester antineoplastic agents into (early/late) endo- or lysosomes, for example, ABCA2-3 [11,26,27], ABCB1 [11,28-30], ABCC1-2 [11,29-31] or ABCG2 [11,29-31]. These transporters were shown to be recruited from the cell membrane surface via internalization into (early/late) endo- or lysosomes, which was demonstrated in colocalization studies with these $\mathrm{ABC}$ transporters and endo- or lysosomal markers, such as early endosomal antigen 1 (EEA1) [11], lysosomal-associated membrane protein 1 (LAMP1) [27], LAMP2 [11,27-29], Lysotracker Blue ${ }^{\circledR}[29]$, Lysotracker Green ${ }^{\circledR}[11]$, Lysotracker Red ${ }^{\circledR}{ }_{[11,27]}$, cathepsin D [11,28,30] or synaptotagmin VII [30]. This is in agreement with colocalizations of other endo- or lysosomal markers, such as caveolin 1 [14], CD63 [12], Nieman-Pick type C [21], RhoB-GFP [22], Rab4 [19], Rab5 [14,19,21], Rab9 [19], Rab11 [19,21], ribophorin2 [32], surfactant protein $C$ [12] or syntaxin 7 [15], with other $A B C$ transporters (e.g., ABCA1 [12], ABCA4-5 [14,15], ABCC5 [19], ABCC8 [32], ABCG1 [21,22] or ABCG4 [22]).

The often stress-induced $[11,28,33]$ sequestration of cytotoxins into (intracellular or -luminal) vesicles serves two main objectives: containment, where the respective antineoplastic agent is safely contained away from its cellular target, thereby preventing its harm to cellular functions, which ultimately results in (cancer) cell survival; and disposal, where vesicles containing cytotoxic agents are processed to the cell surface to form either (multivesicular) lamellar [27] or barrier bodies [11,29], or may be segregated to constitute extracellular vesicles [31,34] before phagocytic processing including degradation of the contained harmful toxins [29]. Extracellular vesicles such as exosomes have also been associated with other $\mathrm{ABC}$ transporters that are not directly linked to vesicle-mediated MDR (e.g., $\mathrm{ABCB} 6$ [35]). Interestingly, $\mathrm{ABC}$ transporters themselves may also serve as cargo in extracellular vesicles. This 
was shown to lead to a donor-to-recipient passing of transport proteins which resulted in an inherited MDR of previously sensitive cells (e.g., ABCB1 [29] or ABCC1 [36]).

Concerning $\mathrm{AD}$, the safe containment of $\mathrm{A} \beta$ proteins in lysosomal compartments and subsequent degradation and/or phagocytic (microglial) processing is vital for cerebral $A \beta$ protein clearance [3,37-39]. Indeed, a disorder in lyso- and autophagosome formation and processing in AD has been described [40,41]. Although there is no direct link between vesicular $A B C$ transporters and lysosomal $A \beta$ storage, it must be considered that several $A B C$ transporters connected to (vesicular) lipid raft composition, organization of (organellar) membrane bilayer microdomains and (cerebral) cholesterol homeostasis, such as ABCA1-2, ABCA5, ABCA7, ABCG1-2 and ABCG4, are highly associated with the emergence and progression of $\mathrm{AD}$ [3]. Interestingly, most of these $\mathrm{ABC}$ transporters were reported to reside in endo- or lysosomal compartments [11,12,15,21,22,26,30,31], and certain ABC transporters were generally associated with phago- and endocytosis as well as autophagy, such as ABCB1 [28,29] or ABCF1 [25]. This implies an important role of $\mathrm{ABC}$ transporters in the degradation and recycling of (sub)cellular components and (macro)molecules. Hence, considering the contribution of $\mathrm{ABC}$ transporters to the formation, trafficking and endo- (as well as exo-)cytosis of intra- (and extra-)cellular vesicles, as well as the formation and establishing of lipid raft microdomains as (immune) response against $\mathrm{A} \beta$ protein excess (so-called 'inflammarafts') [42], a contribution to the emergence and development of $\mathrm{AD}$ by dysfunctional and/or defective vesicular $\mathrm{ABC}$ transporters seems likely.

This gains importance as lipid raft formation and membrane fluidity are major contributors to the formation of (functional) oligomeric $\mathrm{ABC}$ transporter structures. This does not only account for the so-called 'half-transporters', such as ABCB2-3 [3,17,35], ABCB6-10 [3,17,35] and ABCC12-13, as well as the D and G subclass ABC transporters [2,9,20-22], for which a homo- (or hetero- [20]) dimerization is proposed. But also so-called 'full-transporters' were reported to dimerize (e.g., ABCB1 [43], ABCC1 [44] or ABCC9 [45]), and the 'half-transporter' ABCG2 was reported to agglomerate to a tetramer [46], which equals a 'full-transporter'-dimer. Hence, oligomerization to form $\mathrm{ABC}$ transporter units is vital for their function and highly dependent on membrane fluidity.

\section{Implications for drug design \& development: multitarget approaches to uncover black spots}

The discovery of new small molecules to affect underexplored transporters can be challenging. For example, the A subclass $A B C$ transporters comprise of representatives highly associated with $A D$ [3]. Especially dysfunction of $\mathrm{ABCA1}$ and $\mathrm{ABCA7}$ are highly connected to the development and progression of $\mathrm{AD}$ [3]. In fact, $\mathrm{ABCA} 7$ was identified as the second loci showing highest odds ratio for increased AD risk (1.23), just after APOE [47]. However, there are only very few directly interacting small-molecule modulators of these transporters [48,49]. Considering the existence of transporters with no knowledge about their structure, function or interfering small molecules, one is confronted with the question: How can a potent and selective modulator be developed to intervene with the function of such an $\mathrm{ABC}$ transporter?

Fortunately, there are several options to answer this question. The first option is the use of structure-based information: recently, the cryo-electron microscopy (cryo-EM) structures of ABCA1 [50,51] and ABCG2 [52] have been reported, which may be utilized to gain (virtual) information of other $A B C$ transporters of the same subgroup by, for example, homology models. From this structural data, conclusions can be drawn for future drug design and development of small-molecule modulators. With scientific progress, more structural data of $\mathrm{ABC}$ transporters will be reported and available for computational analysis, and hence, the (re)evaluation of (existing) computational models. This will facilitate for more accurate extrapolation and prediction of structural information to unexplored $A B C$ transporters. The second option is the use of ligand-based information: biological data of $A B C$ transporter modulators can be used and transferred to transporter subgroup relatives - or even for their counterparts in other subgroups [53]. This may seem counterintuitive, as the sequence identities (and similarities) of nonclosely related transporters of different subgroups are rather low. However, it has already been shown for broad-spectrum ABCB1, ABCC1 and ABCG2 inhibitors [54-57] and reversers of ABCB1-, ABCC1- and ABCG2-mediated MDR [54-56], that a cross-family modulation of $\mathrm{ABC}$ transporters is possible with one single molecule. This is a striking fact, as the sequence identity (similarity) of these three MDR-conferring transporters is rather low according to sequence analyses [ABCB1, ABCC1: 19.2\% (34.8\%); ABCB1, ABCG2: 13.6\% (23.2\%); ABCC1, ABCG2: 9.2\% (15.6\%)]. Consequently, it can be assumed that common amino acid sequences and structural motifs within the transporters exist beyond the highly conserved nucleotide-binding domains (NBDs). These sequences and motifs may represent a common structural or functional similarity and could be harnessed for drug design and development [53]. This also raises the question if the phylogenetic categorization of $\mathrm{ABC}$ transporters is still contemporary considering their functional similarities. 
Technically, multitarget modulation of $\mathrm{ABC}$ transporters has until now only been described for inhibitors[54-57], which potentially qualifies them for application in cancer-related MDR [1]. However, in AD, these inhibitors may serve as template structures for $\mathrm{ABC}$ transporter activation as a potential treatment option against $\mathrm{AD}$ development and/or progression, since certain compound classes were shown to comprise of both inhibitors and activators [58,59]. Hence, considering all data available on (multitarget) modulators of ABC transporters [1], analysis of the biological data and its correlation to functional substructures of the respective molecule(s), as well as identification of molecular components as indicators for broad-spectrum interaction [53], novel nonselective (promiscuous) activators might be found. This strategy can be supported by computer-based approaches, such as substructure or similarity search, pharmacophore modeling or molecular docking, which already led to the discovery of novel pan-ABC transporter inhibitors [55,57]. Nonselective (promiscuous) activators may be further optimized for selectivity regarding the desired transporter by organic synthesis. On the other hand, selectivity might not even be necessary, as a simultaneous attack on multiple (sub)cellular targets allows for interruption or potentiation of several pathways, and hence, may offer better treatment options [1].

\section{Vesicular ABC transporters: implications for pharmacokinetics}

Promiscuity of molecules could be the first step in the development of novel, highly potent and selective modulators of $\mathrm{ABC}$ transporters that were previously unable to be targeted by small molecules. However, when it comes to target localization, diseases like cancer-related MDR or AD have an additional pharmacokinetic dimension, where the distribution and targeting of small-molecule agents to their (sub)cellular target(s) can be a huge challenge. Nevertheless, targeting of subcellular ABC transporters has already successfully been demonstrated $[11,28]$. Several studies have shown that designing ABCB1 substrates that are lysosomotropic (prone to lysosomal trapping) can induce lysosomal membrane permeabilization (LMP) and cancer cell death by generation of reactive oxygen species (ROS) inside the lysosomes [11,28]. In this instance, the small molecule can use the ABC transporter to its advantage to increase its concentration within lysosomes to exert its lethal effect on cancer cells. Considering that nearly all $\mathrm{ABC}$ transporters contribute to the MDR phenotype [2] and many representatives are functionally active in intracellular vesicular systems [11,26-28,30,31], the above described targeting strategy $[11,28]$ may serve as template to be utilized in conjunction with other $\mathrm{ABC}$ transporters.

Again, the situation in $\mathrm{AD}$ is more complex, as substrates of $\mathrm{ABC}$ transporters may either already fail to cross the $\mathrm{BBB}$ or fail to be retained in the brain. In this case, these agents will never reach their intended subcellular target(s). Such promiscuous or selective substrates would be of great use as AD diagnostics [e.g., positron emission tomography (PET) tracers] to track $\mathrm{ABC}$ transporter function at the $\mathrm{BBB}$ itself [60] (e.g., $\mathrm{ABCB} 1$ [61,62] or $A B C C 1[63,64])$. However, for future drug design of $A D$ therapeutics to affect $A B C$ transporters participating in (subcellular) vesicle membrane formation and composition, these compounds should rather not be substrates but allosteric modulators. As modulation would most likely be conferred through the inter-membrane space, the compounds should be rather lipophilic. This would also be of use when focusing on lipid raft microdomains as potential drug targets [42].

Besides, the oligomerization of 'half-' and 'full-transporters' [43-46] generates new interaction sites, therefore target options for interfering drugs. These sites contain certain motifs and/or domains for vesicular targeting and/or anchorage of $A B C$ transporters (e.g., A subclass ABC transporters [12], ABCB6 [65], ABCD1-3 [20,65] or ABCG1 [21]). Finally, it must be noted that the lipid raft microdomain organization, but also the movement of intracellular $\mathrm{ABC}$ transporter-containing vesicles, is orchestrated by the cytoskeleton [11], which for its part was demonstrated to be influenced by $\mathrm{ABC}$ transporter function (e.g., ABCA1 [42]). In summary, besides ABC transporters themselves, the vesicles in which they reside, the lipid raft microdomains in which they are organized and oligomerized as well as the cytoskeleton are new potential drug targets.

\section{Pan-targets \& pan-agents: concluding remarks}

Awareness of the target and its localization is key for future drug development, which applies to cancer-related $\mathrm{MDR}, \mathrm{AD}$ and other diseases with $\mathrm{ABC}$ transporter participation (e.g., atherosclerosis). Within the last 40 years, a comprehensive knowledge regarding $\mathrm{ABC}$ transporters, their basic functional aspects, as well as interfering small molecules has accumulated [59,66]. However, we still have a limited understanding of $A B C$ transporters, their functional roles, localization sites and specific involvement in disease development and progression. Many ABC transporters have barely been studied, especially in the A and G subfamily. For future target identification and drug design, one must be aware that certain aspects of particular $\mathrm{ABC}$ transporters, such as function and localization, may 
also apply for their subgroup relatives - or even for their non-subgroup counterparts. For example, the sequestration of anticancer drugs into lysosomes has been shown for certain ABC transporters of different subgroups [11,26-28,30,31]. However, the capability of a cell to develop cross-resistance might not be limited to the expression of these particular $\mathrm{ABC}$ transporters but could rather be transferred to other understudied ABC transport proteins as well. These may act solely or in association with other transporters, either functionally [1,2] or via a regulatory interrelationship [1], as already proven for certain representatives $[1,3]$. Moreover, ABC transporters may have a critical contribution to lipid raft microdomain composition and organization, which might not only be limited to (phospho)lipid and cholesterol transporters, which offers a new perspective on target identification in $\mathrm{AD}$ as well. Functional aspects that were formerly perceived as exclusive to a small group of $\mathrm{ABC}$ transporters may be eligible for many other $\mathrm{ABC}$ transporters as well, especially regarding their localization and function in subcellular organelles as well as membrane vesicles. From this, a general intracellular function of these ABC transporters could be concluded, questioning the widely accepted legitimacy of the phylogenetic categorization of $\mathrm{ABC}$ transporters. In all, this seems to be a major challenge for the development and application of novel interacting agents. On the other hand, this complementary information provides a unique opportunity to obtain small molecules as novel and innovative interactors of these understudied targets, to uncover their impact in human diseases and reveal critical pathological aspects. Appreciation of these aspects will truly guide future drug discovery in $\mathrm{ABC}$ transporters-mediated diseases.

Financial \& competing interests disclosure

LYW Leck would like to thank the Australian Research Training Program award and the Australian Rotary Health PhD Scholarship top-up award. PJ Riss is supported by the Norges Forskningsrådet (NFR FRIPRO, Norway; ES231553). The work of J Pahnke was supported by the Deutsche Forschungsgemeinschaft (DFG, German Research Foundation; PA930/9 and PA930/12), the Ministerium für Wirtschaft und Wissenschaft Sachen-Anhalt (Germany; ZS/2016/05/78617), the Leibniz Gemeinschaft (Germany; SAW-2015-IPB-2), the Latvian Council of Science (Latvia; Izp-2018/1-0275), the Nasjonalforeningen (Norway; 16154), HelseS $\varnothing$ (Norway; 2016062, 2019054 and 2019055), the NFR (Norway; PETABC, 251290, 260786), as well as the European Commission (European Union; 643417) and the EEA and Norge Grants TARIMAD (Iceland, Liechtenstein and Norway; TO100078). Furthermore, this project was supported by a project grant from the National Health and Medical Research Council of Australia (NHMRC; 1144456) to PJ Jansson. In addition, PJ Jansson is grateful for his Cancer Institute of New South Wales Career Development Fellowship (CDF171147) and National Breast Cancer Foundation (NBCF) grant support (IIRS-19-048). SMS receives a Benjamin Walter fellowship of the Deutsche Forschungsgemeinschaft (DFG, German Research Foundation; STE2931/2). The authors have no other relevant affiliations or financial involvement with any organization or entity with a financial interest in or financial conflict with the subject matter or materials discussed in the manuscript apart from those disclosed.

No writing assistance was utilized in the production of this manuscript.

\section{Open access}

This work is licensed under the Attribution-NonCommercial-NoDerivatives 4.0 Unported License. To view a copy of this license, visit http://creativecommons.org/licenses/by-nc-nd/4.0/

\section{References}

Papers of special note have been highlighted as: $\bullet$ of interest

1. Stefan SM. Multi-target ABC transporter modulators: what next and where to go? Future Med. Chem. 11(18), 2353-2358 (2019).

- Very first opinion article highlighting the importance of multitarget ATP-binding cassette (ABC) transporter modulators.

2. Pan ST, Li ZL, He ZX, Qiu JX, Zhou SF. Molecular mechanisms for tumour resistance to chemotherapy. Clin. Exp. Pharmacol. Physiol. 43(8), 723-737 (2016).

3. Pereira CD, Martins F, Wiltfang J, da Cruz e Silva OAB, Rebelo S. ABC transportes are key players in Alzheimer's disease. J. Alzheimers Dis. 61(2), 463-485 (2018).

- Most recent article emphasizing the importance of $\mathrm{ABC}$ transporters in Alzheimer's disease.

4. Pahnke J, Walker LC, Scheffler K, Krohn M. Alzheimer's disease and blood-brain barrier function-Why have anti-beta-amyloid therapies failed to prevent dementia progression? Neurosci. Biobehav. Rev. 33(7), 1099-1108 (2009).

5. Pahnke J, Wolkenhauer O, Krohn M, Walker LC. Clinico-pathologic function of cerebral ABC transporters - implications for the pathogenesis of Alzheimer's disease. Curr. Alzheimer. Res. 5(4), 396-405 (2008).

6. Vogelgesang S, Warzok RW, Cascorbi I et al. The role of P-glycoprotein in cerebral amyloid angiopathy; implications for the early pathogenesis of Alzheimer's disease. Curr. Alzheimer. Res. 1(2), 121-125 (2004). 
7. Vogelgesang S, Cascorbi I, Schroeder E et al. Deposition of Alzheimer's beta-amyloid is inversely correlated with P-glycoprotein expression in the brains of elderly non-demented humans. Pharmacogenetics 12(7), 535-541 (2002).

8. Di Martino MT, Scionti F, Sestito $S$ et al. Genetic variants associated with gastrointestinal symptoms in Fabry disease. Oncotarget 7(52), 85895-85904 (2016).

9. Yamanashi Y, Takada T, Yoshikado T, Shoda JI, Suzuki H. NPC2 regulates bilary cholesterol secretion via stimulation of ABCG5/G8-mediated cholesterol transport. Gastroenterology 140(5), 1664-1674 (2011).

10. Zhang M, Sun M, Dwyer NK et al. Differential trafficking of the Niemann-Pick C1 and C2 proteins highlights distinct roles in late endocytic lipid trafficking. Acta Paediatr. Suppl. 91(443), 63-73 (2003).

11. Stefan SM, Jansson PJ, Kalinowski DS, Anjum R, Dharmasivam M, Richardson DR. The growing evidence for targeting P-glycoprotein in lysosomes to overcome resistance. Future Med. Chem 12(6), 473-477 (2020).

- Very first opinion article emphasizing lysosomal $\mathrm{ABC}$ transporters as mediators of multidrug resistance.

12. Beers MF, Hawkins A, Shuman $\mathrm{H}$ et al. A novel conserved targeting motif found in ABCA transporters mediates trafficking to early post-Golgi compartments. J. Lipid Res. 52(8), 1471-1482 (2011).

13. Harris MT, Hussain SS, Inouye CM, Castle AM, Castle JD. Reinterpretation of the localization of the ATP binding cassette transporter ABCG1 in insulin-secreting cells and insights regarding its trafficking and function. PLoS ONE 13(9), e0198383 (2018).

14. Lenis TL, Hu J, Ng SY et al. Expression of ABCA4 in the retinal pigment epithelium and its implications for Stargardt macular degeneration. Proc. Natl Acad. Sci. USA 115(47), E11120-E11127 (2018).

15. Kubo Y, Sekiya S, Ohigashi M et al. ABCA5 resides in lysosomes, and ABCA5 knockout mice develop lysosomal disease-like symptoms. Mol. Cell. Biol. 25(10), 4138-4149 (2005).

16. Kiss K, Kucsma N, Brozik A et al. Role of the N-terminal transmembrane domain in the endo-lysosomal targeting and function of the human ABCB6 protein. Biochem. J. 467(1), 127-139 (2015).

17. Herget M, Tampé R. Intracellular peptide transportes in human - compartmentalization of the "peptidome". Pflugers Arch. 453(5), 591-600 (2007).

18. Hayashi H, Naoi S, Nakagawa T et al. Sorting nexin 27 interacts with multidrug resistance-associated protein 4 (MRP4) and mediates internalization of MRP4. J. Biol. Chem. 287(18), 15054-15065 (2012).

19. Korolnek T, Zhang J, Beardsley S, Scheffer GL, Hamza I. Control of metazoan heme homeostasis by a conserved multidrug resistance protein. Cell Metab. 19(6), 1008-1019 (2014).

20. Kawaguchi K, Morita M. ABC transporter subfamily D: distinct differences in behavior between ABCD1-3 and ABCD4 in subcellular localization, function, and human disease. Biomed. Res. Int. 2006, 6786245 (2016).

21. Tarling EJ, Wdwards PA. ATP binding cassette transporter G1 (ABCG1) is an intracellular sterol transporter. Proc. Natl Acad. Sci. USA 108(49), 19719-19724 (2011).

22. Tarr PT, Edwards PA. ABCG1 and ABCG4 are coexpressed in neurons and astrocytes of the CNS and regulate cholesterol homeostasis through SREBP-2. J. Lipid Res. 49(1), 169-182 (2008).

23. Jungsuwadee P, Nithipongvanitch R, Chen Y et al. MRP1 localization and function in cardiac mitochondria after doxorubicin. Mol. Pharmacol. 75(5), 1117-1126 (2009).

24. Martin LJ, Lau E, Singh H et al. ABCC6 localizes to the mitochondria-associated membrane. Circ. Res. 111(5), 516-520 (2012).

25. Guo F, Ding Y, Caberoy N et al. ABCF1 extrinsically regulates retinal pigment epithelial cell phagocytosis. Mol. Biol. Cell 26(12), 2311-2320 (2015).

26. Aberuyi N, Rahgozar S, Moafi A. The role of ATP-binding cassette transporter A2 in childhood acute lymphoblastic leukemia multidrug resistance. Iran. J. Ped. Hematol. Oncol. 4(3), 118-126 (2014).

27. Chapuy B, Koch R, Radunsky U et al. Intracellular ABC transporter A3 confers multidrug resistance in leukemia cells by lysosomal drug sequestration. Leukemia 22(8), 1576-1586 (2008).

28. Al-Akra L, Bae DH, Sahni $\mathrm{S}$ et al. Tumor stressors induce two mechanisms of intracellular P-glycoprotein-mediated resistance that are overcome by lysosomal-targeted thiosemicarbazones. J. Biol. Chem. 293(10), 3562-3587 (2018).

- Significant analysis of mechanism of stress-induced $\mathrm{ABCB} 1$ internalization and targeting of subcellular $\mathrm{ABC}$ transporter-containing vesicles.

29. Noack A, Gericke B, von Köckritz-Blickwede M et al. Mechanism of drug extrusion by brain endothelial cells via lysosomal drug trapping and disposal by neutrophils. Proc. Natl Acad. Sci. USA 115(41), E9590-9599 (2018). PMID: 30254169

- Demonstration of extracellular phagocytosis and disposal of $\mathrm{ABC}$ transporters-containing vesicles with sequestered and exocytozed xenobiotic agents.

30. Rajagopal A, Simon SM. Subcellular localization and activity of multidrug resistance proteins. Mol. Biol. Cell 14(8), 3389-3399 (2003).

31. Ifergan I, Scheffer GL, Assaraf YG. Novel extracellular vesicles mediate an ABCG2-dependent anticancer drug sequestration resistance. Cancer Res. 65(23), 10952-10958 (2005). 
32. Bao L, Hadjiolova K, Coetzee WA, Rindler MJ. Endosomal KATP channels as a reservoir after myocardial ischemia: a role for SUR2 subunit. Am. J. Physiol. Heart Circ. Physiol. 300(1), H262-H270 (2011).

33. Pérez LM, Milkiewicz P, Elias E, Coleman R, Sánchez Pozzi EJ, Roma MG. Oxidative stress induces internalization of the bile salt export pump, BSEP, and bile salt secretory failure in isolated rat hepatocyte couplets: a role for protein kinase $\mathrm{C}$ and prevention by protein kinase A. Toxicol. Sci. 91(1), 150-158 (2006).

34. Aung T, Chapuy B, Vogel D et al. Exosomal evasion of humoral immunitherapy in aggresive B-cell lymphoma modulated by ATP-binding cassette transporter A3. Proc. Natl Acad. Sci. USA 108(37), 15336-15341 (2011).

35. Kiss K, Brozik A, Kucsma N et al. Shifting the paradigm: the putative mitochondrial protein ABCB6 resides in the lysosomes of cells and in the plasma membrane of erythrocytes. PLoS ONE 7(5), e37378 (2012).

36. Bouvy C, Wannez A, Laloy J, Chatelain C, Dogné JM. Transfer of multidrug resistance among acute myeloid leukemia cells via extracellular vesicles and their microRNA cargo. Leuk. Res. 62, 70-76 (2017).

37. Liu RQ, Zhou QH, Ji SR et al. Membrane localization of beta-amyloid 1-42 in lysosomes: a possible mechanism for lysosome labilization. J. Biol. Chem. 285(26), 19986-19996 (2010).

38. Hwang J, Estick CM, Ikonne US et al. The role of lysosomes in a broad disease-modifying approach evaluated across transgenic mouse models of Alzheimer's disease and Parkinson's disease and models of mild cognitive impairment. Int. J. Mol. Sci. 20(18), 4432 (2019).

39. Cermak S, Kosicek M, Mladenovic-Djordjevic A. Loss of cathepsin B and L to lysosomal dysfunction, NPC-like cholesterol sequestration and accumulation of the key Alzheimer's proteins. PLoS ONE 11(11), e0167428 (2016).

40. Söllvander S, Nikitidou E, Brolin R. Accumulation of amyloid- $\beta$ by astrocytes results in enlarged endosomes and microvesicule-induced apoptosis of neurons. Mol. Neurodegener. 11(1), 38 (2016)

41. Yang Y, Klionsky DJ. Autophagy and disease: unanswered questions. Cell Death Differ. 27(3), 858-871 (2020).

42. Sviridov D, Mukhamedova N, Miller YI. Lipid rafts as a therapeutic target. J. Lipid Res. 61(5), 687-695 (2020).

43. Poruchynsky MS, Ling V. Detection of oligomeric and monomeric forms of P-glycoprotein in multidrug resistant cells. Biochemistry 33(14), 4163-4174 (1994).

44. Rosenberg MF, Mao Q, Holzenburg A, Ford RC, Deeley RG, Cole SP. The structure of the multidrug resistance protein 1 (MRP1/ABCC1). crystallization and single-particle analysis. J. Biol. Chem. 276(19), 16076-16082 (2001).

45. Löffler-Walz C, Hambrock A, Quast U. Interaction of K(ATP) channel modulators with sulfonylurea receptor SUR2B: implications for tetramer formation and allosteric coupling of subunits. Mol. Pharmacol. 61(2), 407-414 (2002).

46. Xu J, Liu Y, Yang Y, Bates S, Zhang JT. Characterization of oligomeric human half-ABC transporter ATP-binding cassette G2. J. Biol. Chem. 279(19), 19781-19789 (2004).

47. Li H, Karl T, Garner B. Understanding the function of ABCA7 in Alzheimer's disease. Biochem. Soc. Trans. 43(5), 920-923 (2015).

48. Favari E, Zanotti I, Zimetti F, Ronda N, Bernini F, Rothblat GH. Probucol inhibits ABCA1-mediated cellular lipid efflux. Aterioscler. Thromb. Vasc. Biol. 24(12), 2345-2350 (2004).

49. Nieland TJF, Chroni A, Fitzgerald ML et al. Cross-inhibition of SR-BI- and ABCA1-mediated cholesterol transport by the small molecules BLT-4 and glyburide. J. Lipid Res. 45(7), 1256-1265 (2004).

50. Qian H, Zhao X, Cao P, Lei J, Yan N, Gong X. Structure of the human lipid exporter ABCA1. Cell 169(7), 1228-1239 (2017).

51. Nosol K, Romane K, Irobalieva RN et al. Cryo-EM structures reveal distinct mechanisms of inhibition of the human multidrug transporter ABCB1. Proc. Natl Acad. Sci. USAdoi:10.1073/pnas.2010264117 (2020) (Epub ahead of print).

52. Jackson SM, Manolaridis I, Kowal J et al. Structural basis of small-molecule inhibition of human multidrug transporter ABCG2. Nat. Struct. Mol. Biol. 25(4), 333-340 (2018).

53. Feldmann C, Bajorath J. Compounds with multitarget activity: structure-based analysis and machine learning. Future Drug Discov. 2(3), FDD44 (2020).

54. Silbermann K, Li J, Namasivayam V et al. Supersior pyrimidine derivatives as selective ABCG2 inhibitors and broad-spectrum ABCB1, ABCC1, and ABCG2 antagonists. J. Med. Chem. 63(18), 10412-10432 (2020).

- Most recent article about multitarget $\mathrm{ABC}$ transporter inhibitors.

55. Silbermann K, Stefan SM, Elshawadfy R, Namasivayam V, Wiese M. Identification of thienopyridine scaffold as an inhibitor of the ABC transport protein ABCC1 (MRP1) and related transporters using a combined virtual screening approach. J. Med. Chem. 62(9), 4383-4400 (2019).

\section{- Very first successful application of a computer-based approach to gain novel multitarget $A B C$ transporter inhibitors.}

56. Stefan K, Schmitt SM, Wiese M. 9-Deazapurines as broad-spectrum inhibitors of the ABC transport proteins P-glycoprotein, multidrug resistance-associated protein 1, and breast cancer resistance protein. J. Med. Chem. 60(21), 8758-8780 (2017).

57. Silbermann K, Li J, Namasivayam V, Stefan SM, Wiese M. Rational drug design of 6-substituted-4-anilino-2-phenylpyrimidines for exploration of novel ABCG2 binding site. Unpublished results under review. 
58. Schmitt SM, Stefan K, Wiese M. Pyrrolopyrimidine derivatives and purine analogs as novel activators of multidrug resistance-associated protein 1 (MRP1, ABCC1). Biochim. Biophys. Acta Biomembr. 1859(1), 69-79 (2017).

59. Wiese M, Stefan SM. The A-B-C of small-molecule ABC transport protein modulators: from inhibition to activation - a case study of multidrug resistance-associated protein 1 (ABCC1). Med. Res. Rev. 39(6), 2031-2081 (2019).

- Very first review article on activators of $A B C$ transport proteins.

60. Kramer V, Brooks AF, Haeger A et al. Evaluation of $\left[{ }^{18} \mathrm{~F}\right]-\mathrm{N}$-methyl iansoprazole as a Tau PET imaging agent in first-in-human studies. ACS Chem. Neurosci. 11(3), 427-435 (2020).

61. Zoufal V, Mairinger S, Brackhan M et al. Imaging P-glycoprotein induction at the blood-brain barrier of a $\beta$-amyloidosis mouse model with ${ }^{11}$ C-metoclopramide PET. J. Nucl. Med. 61(7), 1050-1057 (2020).

62. Zoufal V, Wanek T, Krohn M et al. Age dependency of cerebral P-glycoprotein function in wild-type and APPPS1 mice measured with PET. J. Cereb. Blood Flow Metab. 40(1), 150-162 (2020).

63. Zoufal V, Mairinger S, Krohn M et al. Measurement of cerebral ABCC1 transport activity in wild-type and APP/PS1-21 mice with positron emission tomography. J. Cereb. Blood Flow Metab. 40(5), 954-965 (2020).

64. Zoufal V, Mairinger S, Krohn M et al. Influence of multidrug resistance-associated proteins on the excretion of the ABCC1 imaging probe 6-Bromo-7- $\left[{ }^{11} \mathrm{C}\right]$ methylpurine in mice. Mol. Imaging Biol. 2(2), 306-316 (2019).

65. Kawaguchi K, Okamoto T, Morita M, Imanaka T. Translocation of the ABC transporter ABCD4 from the endoplasmic reticuum to lysosomes requires the escort protein LMBD1. Sci. Rep. 6, 30183 (2016).

66. Stefan SM, Wiese M. Small-molecule inhibitors of multidrug resistance-associated protein 1 and related processes: a historic approach and recent advances. Med. Res. Rev. 39(1), 176-264 (2019). 